Pacific Journal of Mathematics

ON THE DIRICHLET PROBLEM FOR CERTAIN HIGHER 


\title{
ON THE DIRICHLET PROBLEM FOR CERTAIN HIGHER ORDER PARABOLIC EQUATIONS
}

\author{
R. K. JUBERG
}

The Dirichlet problem for the particular equation

$$
D_{x}^{4} u+D_{t} u=0
$$

$\left(D_{\xi} \equiv \partial / \partial \xi\right)$ on the space-time cylinder $(0<x<1) \otimes(0<t \leq T)$ is treated in this paper. However the procedure is directly applicable to the equation $D_{x}^{2 n} u+(-1)^{n} D_{t} u=0$ without technical difficulty and, hence, to any equation simply reducible to this type. It can be applied as well to problems other than the Dirichlet problem. Recently P. G. Kirmser [2] made use of it in solving other interesting problems posed for the equation $D_{x}^{4} u+D_{t} u=0$. There is also an 'uniqueness theorem' contained in his paper.

Using the methods of potential theory, as in Gevrey [1] and Tykhonov [6] for the heat equation and Zeragiya [7] for general second order equations, the problem is reduced to solving a system of integral equations. The integral equations and the integration of them are of interest in themselves.

The procedure affords information on the behavior of the solution along $x=0$ and $x=1$. In addition, the solution obtained allows an analysis of its behavior as $(x, t)$ approaches $(0,0)$ or $(0,1)$ as in the case of the heat equation.

1. Statement of the problem. The problem we pose is to find a function $u(x, t)$ such that

(i) $D_{x} u+D_{t} u=0, \quad 0<x<1, \quad 0<t \leq T$;

(ii) $u(x, 0)=0,0<x<1$;

(iii) $u(0, t)=a(t), \quad u(1, t)=b(t), \quad 0<t \leq T$;

(iv) $\quad D_{x} u(0, t)=c(t), \quad D_{x} u(1, t)=d(t), \quad 0<t \leq T$

where, $a, b, c$, and $d$ are arbitrary functions from classes that we shall presently define. Certain integral operators arise which make it natural to make the following definitions:

DEFINITION 1. Let $S_{1}$ denote the class of functions defined on $(0, T]$ such that to each function, $f(t)$, there corresponds a pair of positive numbers $(\varepsilon, \lambda)$ so that

Received September 9, 1958, and in revised form November 3, 1959. This paper was sponsored by the Office of Naval Research, Contract NONR 710 (16). 


$$
|f|_{1} \equiv \sup _{t, \tau}\left\{\sigma^{\lambda} \frac{|f(t)-f(\tau)|}{|t-\tau|^{\varepsilon}}\right\}<+\infty
$$

where $\sigma=\min (t, \tau), \varepsilon+1 / 4 \leq \lambda<1$.

Definition 2. Let $S_{2}$ denote the class of all functions, $g(t)$, defined on $(0, T]$ and satisfying the conditions:

(i) $g$ uniformly $(\varepsilon+1 / 4)$ - Hölder continuous on any closed subinterval of $(0, T]$, i.e., to each $t_{0} \in(0, T]$ there corresponds a constant $\boldsymbol{c}\left(t_{0}\right)$, depending only on $t_{0}$, such that

$$
\left|g\left(t_{1}\right)-g\left(t_{2}\right)\right| \leq c\left(t_{0}\right)\left|t_{1}-t_{2}\right|^{8+1 / 4}
$$

for all $t_{1}, t_{2} \in\left[t_{0}, T\right]$;

(ii)

$$
\begin{aligned}
|g|_{2} \equiv \sup _{t, \tau}\left\{\sigma^{\lambda} \frac{\mid 4 t^{-1 / 4} g(t)+\int_{0}^{t}[g(t)-g(s)](t-s)^{-5 / 4} d s}{|t-\tau|^{\varepsilon}} \mid\right. \\
\left.\mid \frac{-4 \tau^{-1 / 4} g(\tau)-\int_{0}^{\tau}[g(\tau)-g(s)](\tau-s)^{-5 / 4} d s \mid}{}\right\} \\
\quad+\sup _{t} t^{\lambda-1 / 4}|g(t)|<+\infty
\end{aligned}
$$

where $\sigma, \lambda$, and $\varepsilon$ are as in Definition 1 .

We shall establish existence of solutions to (1.1) for $a, b \in S_{2}$ and $c, d \in S_{1}$.

2. Derivation of the integral equations. By the standard Fourier transform techniques we find the fundamental solution:

$$
k(x-y, t-\tau)=\frac{1}{2 \pi} \int_{-\infty}^{\infty} e^{-i \xi(x-y)} e^{-\xi^{4}(t-\tau)} d \xi, \quad 0 \leq \tau<t
$$

which satisfies

$$
D_{x}^{4} k+D_{t} k=0
$$

and

$$
D_{y}^{4} k-D_{\tau} k=0 \text {. }
$$

In the sequel we will frequently use the following basic estimates of the fundamental solution and of derivatives of same due to 0 . Ladyzhenskaya [3] (see also P. C. Rosenbloom [5]):

$$
\left|D_{x}^{\nu} k(x, t)\right| \leq c_{1}(\nu) \cdot t^{-(1+\nu) / 4} \cdot \exp \left[-c_{2}\left(x^{4} / t\right)^{1 / 3}\right]
$$

\footnotetext{
${ }^{1}$ See appendix.
} 
where $D_{x}^{\nu} \equiv(\partial / \partial x)^{\nu}, c_{1}$ depends on $\nu$, and $c_{2}$ is an absolute constant.

\section{LEMMA 1.}

(2.3) $p(x)-\int_{0}^{1} k(x-y, t) d y=\int_{0}^{t}\left[D_{y}^{3} k(x-1, t-\sigma)-D_{y}^{3} k(x, t-\sigma)\right] d \sigma$ where

$$
p(x)= \begin{cases}1, & 0<x<1 \\ \frac{1}{2}, & x=0, x=1 \\ 0, & x<0, x>1 .\end{cases}
$$

Proof. Since $D_{\sigma} k=D_{y} k$,

$$
\begin{aligned}
\int_{0}^{1} D_{\sigma} k(x-y, t-\sigma) d y & =\int_{0}^{1} D_{y}^{4} k(x-y, t-\sigma) d y=D_{y}^{3} k(x-y, t-\sigma) \mid \begin{array}{l}
y=1 \\
y=0
\end{array} \\
& =D_{y}^{3} k(x-1, t-\sigma)-D_{y}^{3} k(x, t-\sigma) .
\end{aligned}
$$

Integrating with respect to $\sigma$ from 0 to $t-\varepsilon$ gives

$$
\begin{aligned}
& \int_{0}^{t-\varepsilon}\left(\int_{0}^{1} D_{\sigma} k(x-y, t-\sigma) d y\right) d \sigma=\int_{0}^{t-\varepsilon} D_{\sigma}\left(\int_{0}^{1} k(x-y, t-\sigma) d y\right) d \sigma \\
& \quad=\int_{0}^{1} k(x-y, \varepsilon) d y-\int_{0}^{1} k(x-y, t) d y \\
& \quad=\int_{0}^{t-\varepsilon}\left[D_{y}^{3} k(x-1, t-\sigma)-D_{y}^{3} k(x, t-\sigma] d \sigma .\right.
\end{aligned}
$$

That is,

$$
\begin{aligned}
& \int_{0}^{1} k(x-y, \varepsilon) d y-\int_{0}^{1} k(x-y, t) d y \\
& \quad=\int_{0}^{t-\varepsilon}\left[D_{y}^{3} k(x-1, t-\sigma)-D_{y}^{3} k(x, t-\sigma)\right] d \sigma .
\end{aligned}
$$

Since $k(x-y, \varepsilon)=\varepsilon^{-1 / 4} k\left((x-y) / \varepsilon^{1 / 4}, 1\right)$ and $k(-z, 1)=k(z, 1)$,

$$
\int_{0}^{1} k(x-y, \varepsilon) d y=\int_{0}^{1} k\left((x-y) / \varepsilon^{1 / 4}, 1\right) \frac{d y}{\varepsilon^{1 / 4}}=\int_{-x / \varepsilon^{1 / 4}}^{(1-x) / \varepsilon^{1 / 4}} k(z, 1) d z .
$$

Hence

$$
\lim _{\varepsilon \downarrow 0} \int_{0}^{1} k(x-y, \varepsilon) d y= \begin{cases}0, & x<0, x>1 \\ \int_{-\infty}^{0} k(z, 1) d z, & x=1 \\ \int_{0}^{\infty} k(z, 1) d z, & x=0 \\ \int_{-\infty}^{\infty} k(z, 1) d z, & 0<x<1 .\end{cases}
$$


Now

$$
\begin{aligned}
& \int_{-\infty}^{\infty} k(z, 1) d z=\int_{-\infty}^{\infty}\left(\frac{1}{2 \pi} \int_{-\infty}^{\infty} e^{-i \xi \cdot z} \cdot e^{-\xi^{4}} d \xi\right) d z \\
& =\left.\frac{1}{\sqrt{2 \pi}} \int_{-\infty}^{\infty} e^{i z \cdot w}\left(\frac{1}{\sqrt{2 \pi}} \int_{-\infty}^{\infty} e^{-i \xi \cdot z} \cdot e^{-\xi^{4}} d \xi\right) d z\right|_{w=0}=\left.e^{-w^{4}}\right|_{w=0}=1
\end{aligned}
$$

and

$$
\int_{0}^{\infty} k(z, 1) d z=\int_{-\infty}^{0} k(z, 1) d z=\frac{1}{2} \int_{-\infty}^{\infty} k(z, 1) d z=\frac{1}{2}
$$

Thus,

$$
\begin{aligned}
\int_{0}^{t} & {\left[D_{y}^{3} k(x-1, t-\sigma)-D_{y}^{3} k(x, t-\sigma)\right] d \sigma } \\
& =\lim _{\varepsilon \downarrow 0} \int_{0}^{t-\varepsilon}\left[D_{y}^{3} k(x-1, t-\sigma)-D_{y}^{3} k(x, t-\sigma)\right] d \sigma \\
& =p(x)-\int_{0}^{1} k(x-y, t) d y .
\end{aligned}
$$

In particular, since $D_{y}^{3} k(0, t-\sigma) \equiv 0$

$$
\frac{1}{2}-\int_{0}^{1} k(1-y, t) d y=-\int_{0}^{t} D_{y}^{3} k(1, t-\sigma) d \sigma
$$

and

$$
\frac{1}{2}-\int_{0}^{1} k(-y, t) d y=\int_{0}^{t} D_{y}^{3} k(-1, t-\sigma) d \sigma
$$

However, since $k(-y, t)=k(y, t)$,

$$
D_{y}^{3} k(-1, t-\sigma)=-D_{y}^{3} k(1, t-\sigma),
$$

and

$$
\begin{gathered}
\int_{0}^{1} k(1-y, t) d y=\int_{0}^{1} k(y, t) d y \\
\frac{1}{2}-\int_{0}^{1} k(y, t) d y=-\int_{0}^{t} D_{y}^{3} k(1, t-\sigma) d \sigma .
\end{gathered}
$$

In deriving the integral equations we will need the following limit relations.

Lemma 2.

$$
f \in S_{i}, \quad i=1,2
$$




$$
\lim _{\substack{x<0 \\ 0<x<1}} \int_{0}^{t} f(\sigma) D_{y}^{3} k(x, t-\sigma) d \sigma=-\frac{1}{2} f(t)
$$

$$
\lim _{\substack{x \uparrow 1 \\ 0<x<1}} \int_{0}^{t} f(\sigma) D_{y}^{3} k(x-1, t-\sigma) d \sigma=\frac{1}{2} f(t)
$$

$$
g \in S_{2}
$$

$$
\begin{aligned}
& \lim _{\substack{x \neq 0 \\
0<x<1}} \int_{0}^{t} g(\sigma) D_{y}^{4} k(x, t-\sigma) d \sigma \\
& \quad=-g(t) k(0, t)-\int_{0}^{t}[g(t)-g(\sigma)] D_{\sigma} k(0, t-\sigma) d \sigma \\
& \lim _{\substack{x \uparrow 1 \\
0<x<1}} \int_{0}^{t} g(\sigma) D_{y}^{4} k(x-1, t-\sigma) d \sigma \\
& \quad=-g(t) k(0, t)-\int_{0}^{t}[g(t)-g(\sigma)] D_{\sigma} k(0, t-\sigma) d \sigma .
\end{aligned}
$$

Proof. Part (a). We shall prove (2.5) for $f \in S_{1}$. The proofs for the remaining cases are essentially the same.

We write

$$
\begin{aligned}
& \int_{0}^{t} f(\sigma) D_{y}^{3} k(x, t-\sigma) d \sigma \\
& \quad=f(t) \int_{0}^{t} D_{y}^{3} k(x, t-\sigma) d \sigma-\int_{0}^{t}[f(t)-f(\sigma)] D_{y}^{3} k(x, t-\sigma) d \sigma .
\end{aligned}
$$

From (2.2) and the hypothesis on $f$

$$
\begin{aligned}
|[f(t)-f(\sigma)]| \cdot\left|D_{y}^{3} k(x, t-\sigma)\right| \leq & |f|_{1} \cdot \sigma^{-\lambda}(t-\sigma)^{\varepsilon} \cdot c_{1}(t-\sigma)^{-1} \cdot e^{-c_{2}\left(x^{4} /(t-\sigma)\right)^{1 / 3}} \\
& \leq(\text { constant }) \cdot \sigma^{-\lambda}(t-\sigma)^{\varepsilon-1} .
\end{aligned}
$$

Hence, by the dominated convergence theorem:

$$
\begin{aligned}
\lim _{\substack{x \downarrow 0 \\
0<x<1}} \int_{0}^{t}[f(t)-f(\sigma)] \cdot D_{v}^{3} k(x, t-\sigma) d \sigma \\
\quad=\int_{0}^{t} \lim _{x \downarrow 0}[f(t)-f(\sigma)] \cdot D_{y}^{3} k(x, t-\sigma) d \sigma=0 .
\end{aligned}
$$

Thus

$$
\lim _{x \downarrow 0} \int_{0}^{t} f(\sigma) \cdot D_{y}^{3} k(x, t-\sigma) d \sigma=f(t) \lim _{x \downarrow 0} \int_{0}^{t} D_{y}^{3} k(x, t-\sigma) d \sigma
$$

which by (2.3) equals

$$
f(t) \lim _{x \downarrow 0}\left\{\int_{0}^{t} D_{y}^{3} k(x-1, t-\sigma) d \sigma+\int_{0}^{1} k(x-y, t) d y-1\right\}
$$




$$
=f(t)\left\{\int_{0}^{t} D_{y}^{3} k(-1, t-\sigma) d \sigma+\int_{0}^{1} k(-y, t) d y-1\right\}
$$

and by (2.4) this equals

$$
f(t)\left\{\frac{1}{2}-1\right\}=-\frac{1}{2} f(t) .
$$

Part (b). We shall give the proof of (2.7). As above write

$$
\begin{aligned}
& \int_{0}^{t} g(\sigma) \cdot D_{y}^{4} k(x, t-\sigma) d \sigma \\
& =g(t) \int_{0}^{t} D_{y}^{4} k(x, t-\sigma) d \sigma-\int_{0}^{t}[g(t)-g(\sigma)] D_{y}^{4} k(x, t-\sigma) d \sigma \\
& =g(t) \int_{0}^{t} D_{\sigma} k(x, t-\sigma) d \sigma-\int_{0}^{t}[g(t)-g(\sigma)] D_{\sigma} k(x, t-\sigma) d \sigma \\
& =g(t) \cdot\left\{k(x, t-\sigma) \mid \begin{array}{lll}
\sigma & \uparrow & t \\
\sigma= & =
\end{array}\right\}-\int_{0}^{t}[g(t)-g(\sigma)] \cdot D_{\sigma} k(x, t-\sigma) d \sigma \\
& =-g(t) \cdot k(x, t)-\int_{0}^{t}[g(t)-g(\sigma)] \cdot D_{\sigma} k(x, t-\sigma) d \sigma \\
& =-g(t) \cdot k(x, t)-\int_{0}^{t / 2}[g(t)-g(\sigma)] \cdot D_{\sigma} k(x, t-\sigma) d \sigma \\
& -\int_{t / 2}^{t}[g(t)-g(\sigma)] \cdot D_{\sigma} k(x, t-\sigma) d \sigma \text {. }
\end{aligned}
$$

For given $t \in(0, T]$, the first two terms are continuous in $x$, for all $x$, and the interchange of limit and integration in the latter is justified as above, using the Hölder continuity of $g$. From these remarks, the proof follows.

We seek a solution to our problem in the following form:

$$
\begin{aligned}
& u(x, t)=\int_{0}^{t} \alpha(\sigma) D_{y}^{3} k(x, t-\sigma) d \sigma+\int_{0}^{t} \beta(\sigma) \cdot D_{y}^{3} k(x-1, t-\sigma) d \sigma \\
& +\int_{0}^{t} \gamma(\sigma) \cdot D_{y}^{2} k(x, t-\sigma) d \sigma+\int_{0}^{t} \delta(\sigma) \cdot D_{y}^{2} k(x-1, t-\sigma) d \sigma, 0<x<1
\end{aligned}
$$

where $\alpha, \beta \in S_{2}$ and $\gamma, \delta \in S_{1}$. The fact that $u(x, t)$ satisfies the equation for $0<x<1$ follows from (2.2), which justifies interchanging the order of differentiation and integration, and (2.1). From Lemma 2, we shall obtain a system of integral equations for the unknown functions $\alpha, \beta, \gamma$, and $\delta$.

From (2.5) and (2.6) we obtain, upon taking the limit of (2.9) first as $x \downarrow 0$, and then as $x \uparrow 1$, the equations

$$
\begin{aligned}
a(t)= & -\frac{1}{2} \alpha(t)+\int_{0}^{t} \beta(\sigma) \cdot D_{y}^{3} k(-1, t-\sigma) d \sigma \\
& +\int_{0}^{t} \gamma(\sigma) \cdot D_{y}^{2} k(0, t-\sigma) d \sigma+\int_{0}^{t} \delta(\sigma) \cdot D_{y}^{2} k(-1, t-\sigma) d \sigma
\end{aligned}
$$


and

$$
\begin{aligned}
b(t)= & \int_{0}^{t} \alpha(\sigma) \cdot D_{y}^{3} k(1, t-\sigma) d \sigma+\frac{1}{2} \beta(t) \\
& +\int_{0}^{t} \gamma(\sigma) D_{y}^{2} k(1, t-\sigma) d \sigma+\int_{0}^{t} \delta(\sigma) \cdot D_{y}^{2} k(0, t-\sigma) d \sigma .
\end{aligned}
$$

The limits obtained from the various terms other than those where Lemma 2 is applied are by continuity which follows from (2.2).

Since

$$
D_{y}^{3} k(-1, t-\sigma)=-D_{y}^{3} k(1, t-\sigma)
$$

and

$$
D_{y}^{2} k(-1, t-\sigma)=D_{y}^{2} k(1, t-\sigma),
$$

we can write (2.10) as

$$
\begin{aligned}
a(t)= & -\frac{1}{2} \alpha(t)-\int_{0}^{t} \beta(\sigma) \cdot D_{y}^{3} k(1, t-\sigma) d \sigma \\
& +\int_{0}^{t} \gamma(\sigma) \cdot D_{y}^{2} k(0, t-\sigma) d \sigma+\int_{0}^{t} \delta(\sigma) \cdot D_{y}^{2} k(1, t-\sigma) d \sigma .
\end{aligned}
$$

From (2.1)

$$
\begin{aligned}
D_{x} u(x, t)= & -\int_{0}^{t} \alpha(\sigma) \cdot D_{y}^{4} k(x, t-\sigma) d \sigma-\int_{0}^{t} \beta(\sigma) \cdot D_{y}^{4} k(x-1, t-\sigma) d \sigma \\
& -\int_{0}^{t} \gamma(\sigma) \cdot D_{y}^{3} k(x, t-\sigma) d \sigma-\int_{0}^{t} \delta(\sigma) \cdot D_{y}^{3} k(x-1, t-\sigma) d \sigma .
\end{aligned}
$$

Using (2.7) and (2.8) we obtain upon taking the limit of this relation as $x \downarrow 0$ and then as $x \uparrow 1$, the equations

$$
\begin{aligned}
c(t)= & \alpha(t) \cdot k(0, t)+\int_{0}^{t}[\alpha(t)-\alpha(\sigma)] D_{\sigma} k(0, t-\sigma) d \sigma \\
& -\int_{0}^{t} \beta(\sigma) \cdot D_{\sigma} k(1, t-\sigma) d \sigma+\frac{1}{2} \gamma(t)+\int_{0}^{t} \delta(\sigma) \cdot D_{y}^{3} k(1, t-\sigma) d \sigma
\end{aligned}
$$

and

$$
\begin{aligned}
d(t)= & -\int_{0}^{t} \alpha(\sigma) \cdot D_{\sigma} k(1, t-\sigma) d \sigma+\beta(t) \cdot k(0, t) \\
& +\int_{0}^{t}[\beta(t)-\beta(0)] D_{\sigma} k(0, t-\sigma) d \sigma \\
& -\int_{0}^{t} \gamma(\sigma) \cdot D_{y}^{3} k(1, t-\sigma) d \sigma-\frac{1}{2} \delta(t) .
\end{aligned}
$$

Adding and subtracting (2.10)' and (2.11) gives 


$$
\begin{aligned}
& a(t) \pm b(t)=-\frac{1}{2}[\alpha(t) \mp \beta(t)] \pm \int_{0}^{t}[\alpha(\sigma) \mp \beta(\sigma)] \cdot D_{y}^{3} k(1, t-\sigma) d \sigma \\
& \quad+\int_{0}^{t}[\gamma(\sigma) \pm \delta(\sigma)] D_{v}^{2} k(0, t-\sigma) d \sigma \pm \int_{0}^{t}[\gamma(\sigma) \pm \delta(\sigma)] D_{y}^{2} k(1, t-\sigma) d \sigma .
\end{aligned}
$$

Similarly, adding and subtracting (2.12) and (2.13) gives

$$
\begin{aligned}
c(t) & \pm d(t)=[\alpha(t) \pm \beta(t)] k(0, t) \\
& +\int_{0}^{t}\{[\alpha(t) \pm \beta(t)]-[\alpha(\sigma) \pm \beta(\sigma)]\} D_{\sigma} k(0, t-\sigma) d \sigma \\
& \mp \int_{0}^{t}[\alpha(\sigma) \pm \beta(\sigma)] \cdot D_{\sigma} k(1, t-\sigma) d \sigma \mp \int_{0}^{t}[\gamma(\sigma) \mp \delta(\sigma)] D_{y}^{3} k(1, t-\sigma) d \sigma \\
& +\frac{1}{2}[\gamma(t) \mp \delta(t)] .
\end{aligned}
$$

Setting

$$
\begin{aligned}
\phi(t) & =\gamma(t)+\delta(t) & A(t) & =c(t)-d(t) \\
\psi(t) & =\alpha(t)-\beta(t) & B(t) & =a(t)+b(t) \\
f(t) & =\gamma(t)-\delta(t) & C(t) & =c(t)+d(t) \\
g(t) & =\alpha(t)+\beta(t) & D(t) & =a(t)-b(t)
\end{aligned}
$$

we obtain the following pairs of equations

$$
\begin{aligned}
\frac{1}{2} \phi(t) & +\int_{0}^{t} \phi(\sigma) \cdot D_{y}^{3} k(1, t-\sigma) d \sigma+\psi(t) k(0, t) \\
& +\int_{0}^{t}[\psi(t)-\psi(\sigma)] D_{\sigma} k(0, t-\sigma) d \sigma \\
& +\int_{0}^{t} \psi(\sigma) \cdot D_{\sigma} k(1, t-\sigma) d \sigma=A(t) \\
\int_{0}^{t} \phi(\sigma) \cdot D_{y}^{2} k(0, t-\sigma) d \sigma & +\int_{0}^{t} \phi(\sigma) \cdot D_{y}^{2} k(1, t-\sigma) d \sigma-\frac{1}{2} \psi(t) \\
& +\int_{0}^{t} \psi(\sigma) \cdot D_{y}^{3} k(1, t-\sigma) d \sigma=B(t),
\end{aligned}
$$

and

$$
\begin{aligned}
& \frac{1}{2} f(t)-\int_{0}^{t} f(\sigma) \cdot D_{y}^{3} k(1, t-\sigma) d \sigma+g(t) \cdot k(0, t) \\
&+\int_{0}^{t}[g(t)-g(\sigma)] \cdot D_{\sigma} k(0, t-\sigma) d \sigma \\
&-\int_{0}^{t} g(\sigma) \cdot D_{\sigma} k(1, t-\sigma) d \sigma=C(t) \\
& \int_{0}^{t} f(\sigma) \cdot D_{y}^{2} k(0, t-\sigma) d \sigma-\int_{0}^{t} f(\sigma) \cdot D_{y}^{2} k(1, t-\sigma) d \sigma-\frac{1}{2} g(t)
\end{aligned}
$$




$$
-\int_{0}^{t} g(\sigma) \cdot D_{y}^{3} k(1, t-\sigma) d \sigma=D(t)
$$

3. Solution of the integral equations. To facilitate the solution of the integral equations, we define for suitable functions $f$ and $g$

$$
\left(T_{1} f\right)(t)=\frac{1}{\Gamma(1 / 4)} \int_{0}^{t} f(\sigma)(t-\sigma)^{-3 / 4} d \sigma
$$

and

$$
\left(T_{2} g\right)(t)=-\frac{1}{\Gamma(-1 / 4)}\left[4 t^{-1 / 4} \cdot g(t)+\int_{0}^{t}[g(t)-g(\sigma)](t-\sigma)^{-5 / 4} d \sigma\right]
$$

$T_{1}$ is the operator which is commonly called $I^{1 / 4}$ (see M. Riesz [4]). However, it is not immediately clear that $T_{2}$ is $I^{-1 / 4}$ because of the singularities allowed at the origin in the classes of functions under consideration. The following example will illustrate the effect of the singularity at the origin. Let

$$
h(t)=t^{-1+\delta}, \quad 0<\delta<\frac{1}{4} \quad\left(h \notin S_{2}\right) .
$$

Then

$$
\left(T_{2} h\right)(t)=\frac{\Gamma(\delta)}{\Gamma(\delta-1 / 4)} \cdot t^{-5 / 4+\delta},
$$

a function to which $T_{1}$ (or $I^{1 / 4}$ ) cannot be applied. Using the methods employed by M. Riesz in the theory of Riemann-Liouville integrals, we shall show that on the classes under consideration $T_{2}$ is actually $I^{-1 / 4}$.

THEOREM 1. If $f \in S_{1}$, then $T_{1} f$ is uniformly $(\varepsilon+1 / 4)$-Hölder continuous on any closed subinterval of $(0, T]$ where the $\varepsilon$ is that associated with $f \in S_{1}{ }^{2}$.

Proof. Let $t, \tau \in[\delta, T], \delta>0$. Assume without loss of generality $\tau<t$. Form the difference

$$
\begin{aligned}
\Delta & \equiv \Gamma\left(\frac{1}{4}\right)\left[\left(T_{1} f\right)(t)-\left(T_{1} f\right)(\tau)\right] \\
& =\int_{0}^{t} f(\sigma) \cdot(t-\sigma)^{-3 / 4} d \sigma-\int_{0}^{\tau} f(\sigma) \cdot(\tau-\sigma)^{-3 / 4} d \sigma .
\end{aligned}
$$

Adding and subtracting $f(t)$ in the integrands gives

2 This Theorem is essentially contained in Hardy, G. H. and Littlewood, J. E., "Some properties of fractional integrals", Math. Zeit., Vol 27, (1928), pp. 565-606. 


$$
\begin{aligned}
\Delta= & f(t) \cdot \int_{0}^{t}(t-\sigma)^{-3 / 4} d \sigma \\
& +\int_{0}^{t}[f(\sigma)-f(t)] \cdot(t-\sigma)^{-3 / 4} d \sigma-f(t) \cdot \int_{0}^{\tau}(\tau-\sigma)^{-3 / 4} d \sigma \\
& -\int_{0}^{\tau}[f(\sigma)-g(t)] \cdot(\tau-\sigma)^{-3 / 4} d \sigma=4 f(t)\left(t^{1 / 4}-\tau^{1 / 4}\right) \\
& +\int_{0}^{\tau}[f(\sigma)-f(t)] \cdot\left[(t-\sigma)^{-3 / 4}-(\tau-\sigma)^{-3 / 4}\right] d \sigma \\
& +\int_{\tau}^{t}[f(\sigma)-f(t)] \cdot(t-\sigma)^{-3 / 4} d \sigma \equiv I_{1}+I_{2}+I_{3}
\end{aligned}
$$

say.

Regarding $I_{2}$, write it as

$$
\begin{aligned}
I_{2}= & \int_{0}^{\delta / 2}[f(\sigma)-f(t)]\left[(t-\sigma)^{-3 / 4}-(\tau-\sigma)^{-3 / 4}\right] d \sigma \\
& +\int_{\delta / 2}^{t}[f(\sigma)-f(t)] \cdot\left[(t-\sigma)^{-3 / 4}-(\tau-\sigma)^{-3 / 4}\right] d \sigma \equiv J_{21}+J_{22} \cdot
\end{aligned}
$$

Using the mean-value theorem

$$
\begin{aligned}
\mid f(\sigma)- & f(t) \mid \cdot\left[(\tau-\sigma)^{-3 / 4}-(t-\sigma)^{-3 / 4}\right] \\
& =|f(\sigma)-f(t)| 3 / 4[(\tau-\sigma)+\theta(t-\tau)]^{-7 / 4} \cdot(t-\tau), 0 \leq \theta \leq 1 .
\end{aligned}
$$

Since $t-\sigma, \tau-\sigma \geq \delta / 2$ for $J_{21}$,

$$
\begin{gathered}
|f(\sigma)-f(t)| \cdot\left[(\tau-\sigma)^{-3 / 4}-(t-\sigma)^{-3 / 4}\right] \leq \sigma^{-\lambda}(t-\sigma)^{\varepsilon} \cdot|f|_{1} \cdot 3 / 4 \cdot(\delta / 2)^{-7 / 4} \cdot(t-\tau) \\
\leq 3 / 4 \cdot T^{\varepsilon} \cdot(2 / \delta)^{7 / 4} \cdot|f|_{1} \cdot \sigma^{-\lambda}(t-\tau)=(\text { constant }) \cdot \sigma^{-\lambda} \cdot(t-\tau)
\end{gathered}
$$

Thus,

$$
\begin{aligned}
\left|J_{21}\right| & =\left|\int_{0}^{\delta / 2}[f(\sigma)-f(t)] \cdot\left[(t-\sigma)^{-3 / 4}-(\tau-\sigma)^{-3 / 4}\right] d \sigma\right| \\
& \leq \int_{0}^{\delta / 2}|f(\sigma)-f(t)| \cdot\left[(\tau-\sigma)^{-3 / 4}-(t-\sigma)^{3 / 4}\right] d \sigma \\
& \leq(\text { constant }) \cdot(t-\tau) \int_{0}^{\delta / 2} \sigma^{-\lambda} d \sigma=(\text { constant }) \cdot(t-\tau) .
\end{aligned}
$$

Now

$$
\begin{aligned}
\left|J_{22}\right| & \leq|f|_{1} \cdot \int_{\delta / 2}^{\tau} \sigma^{-\lambda}(t-\sigma)^{\varepsilon} \cdot\left[(\tau-\sigma)^{-3 / 4}-(t-\sigma)^{-3 / 4}\right] d \sigma \\
& \leq(\delta / 2)^{-\lambda} \cdot|f|_{1} \cdot \int_{\delta / 2}^{\tau}(t-\sigma)^{\varepsilon} \cdot\left[(\tau-\sigma)^{-3 / 4}-(t-\sigma)^{-3 / 4}\right] d \sigma
\end{aligned}
$$




$$
\leq(2 / \delta)^{\lambda} \cdot|f|_{1} \cdot \int_{0}^{\tau}(t-\sigma)^{\varepsilon} \cdot\left[(\tau-\sigma)^{-3 / 4}-(t-\sigma)^{-3 / 4}\right] d \sigma .
$$

Set $\tau-\sigma=(t-\sigma) s$. Then

$$
\begin{aligned}
& t-\sigma=\frac{t-\tau}{1-s}, \\
& \tau-\sigma=\frac{s(t-\tau)}{1-s},
\end{aligned}
$$

and

Hence

$$
d \sigma=-\frac{t-\tau}{(1-s)^{2}} d s
$$

$$
\begin{aligned}
\left|J_{22}\right| & \leq(\text { constant })(t-\tau)^{1 / 4+\varepsilon} \cdot \int_{0}^{\tau / t}(1-s)^{-5 / 4-\varepsilon} \cdot\left(s^{-3 / 4}-1\right) d s \\
& \leq(\text { constant })(t-\tau)^{1 / 4+\varepsilon} \cdot \int_{0}^{1}(1-s)^{-5 / 4-\varepsilon} \cdot\left(1-s^{3 / 4}\right) \cdot s^{-3 / 4} d s \\
& \leq(\text { constant }) \cdot(t-\tau)^{1 / 4+\varepsilon}
\end{aligned}
$$

the latter integral existing for $\varepsilon<3 / 4$ since

$$
\begin{aligned}
& (1-s)^{-5 / 4-\varepsilon} \cdot\left(1-s^{3 / 4}\right) \cdot s^{-3 / 4} \\
& \quad=s^{-3 / 4} \cdot(1-s)^{-1 / 4-\varepsilon} \cdot\left(1+s+s^{2}\right)\left(1+s^{3 / 2}\right)^{-1}\left(1+s^{3 / 4}\right)^{-1} .
\end{aligned}
$$

Now

$$
\begin{aligned}
\left|I_{3}\right| & \leq \int_{\tau}^{t}|f(\sigma)-f(t)| \cdot(t-\sigma)^{-3 / 4} d \sigma \leq|f|_{1} \cdot \int_{\tau}^{t} \sigma^{-\lambda}(t-\sigma)^{\varepsilon-3 / 4} d \sigma \\
& \leq(2 / \delta)^{\lambda} \cdot|f|_{1} \cdot \int_{\tau}^{t}(t-\sigma)^{\varepsilon-3 / 4} d \sigma=(\text { constant })(t-\tau)^{\varepsilon+1 / 4}
\end{aligned}
$$

This completes the proof.

Q.E.D.

THEOREM 2. $f \in S_{1}$

(i) $T_{2} T_{1}=I_{1}$, where $I_{1}$ is the identity transformation on $S_{1}$.

(ii) $T_{1} f \in S_{2}$.

Proof.

(i) $\quad\left[T_{2}\left(T_{1} f\right)\right](t)=\frac{-1}{\Gamma(1 / 4) \cdot \Gamma(-1 / 4)}\left\{4 t^{-1 / 4} \int_{0}^{t} f(\sigma)(t-\sigma)^{-3 / 4} d \sigma\right.$

$$
\left.+\int_{0}^{t}\left[\int_{0}^{t} f(\sigma)(t-\sigma)^{-3 / 4} d \sigma-\int_{0}^{\tau} f(\sigma)(\tau-\sigma)^{-3 / 4} d \sigma\right](t-\tau)^{-5 / 4} d \tau\right\}
$$


We proceed as in the theory of Riemann-Liouville integrals. Define

$$
F(\mu)=\frac{1}{\Gamma(\mu)} \int_{0}^{t}\left[\int_{0}^{t} f(\sigma)(t-\sigma)^{-3 / 4} d \sigma-\int_{0}^{\tau} f(\sigma)(\tau-\sigma)^{-3 / 4} d \sigma\right](t-\tau)^{\mu-1} d \tau
$$

which exists and is analytic for $\Re e \mu>-1 / 4-\varepsilon$ by Theorem 1 . Now restrict $\mu$ so that $\Re e \mu>0$. Then

$$
\begin{aligned}
F(\mu)= & \frac{1}{\Gamma(\mu)} \int_{0}^{t} f(\sigma)(t-\sigma)^{-3 / 4} d \sigma \cdot \int_{0}^{t}(t-\tau)^{\mu-1} d \tau \\
& -\frac{1}{\Gamma(\mu)} \int_{0}^{t}\left(\int_{0}^{\tau} f(\sigma)(\tau-\sigma)^{-3 / 4} d \sigma\right)(t-\tau)^{\mu-1} d \tau \\
= & \frac{t^{\mu}}{\mu \Gamma(\mu)} \cdot \int_{0}^{t} f(\sigma) \cdot(t-\sigma)^{-3 / 4} d \sigma-\frac{1}{\Gamma(\mu)} \int_{0}^{t}\left(\int_{0}^{\tau} f(\sigma)(\tau-\sigma)^{-3 / 4} d \sigma\right) \\
& \times(t-\tau)^{\mu-1} d \tau .
\end{aligned}
$$

Interchanging the order of integration in the second term and setting $\tau-\sigma=(t-\sigma) \cdot s$ in the inner integral gives

$$
\begin{aligned}
& \frac{1}{\Gamma(\mu)} \int_{0}^{t} f(\sigma) \cdot(t-\sigma)^{\mu-3 / 4} d \sigma \cdot\left(\int_{0}^{1} s^{-3 / 4}(1-s)^{\mu-1} d s\right) \\
& \quad=\frac{\Gamma(1 / 4)}{\Gamma(\mu+1 / 4)} \cdot \int_{0}^{t} f(\sigma) \cdot(t-\sigma)^{\mu-3 / 4} d \sigma .
\end{aligned}
$$

Adding and subtracting $f(t)$ in the integrand of this latter integral gives

$$
\begin{aligned}
& \frac{\Gamma(1 / 4)}{\Gamma(\mu+1 / 4)} \int_{0}^{t} f(\sigma) \cdot(t-\sigma)^{\mu-3 / 4} d \sigma=\frac{\Gamma(1 / 4) \cdot f(t)}{\Gamma(\mu+1 / 4)} \cdot \int_{0}^{t}(t-\sigma)^{\mu-3 / 4} d \sigma \\
& \quad+\frac{\Gamma(1 / 4)}{\Gamma(\mu+1 / 4)} \int_{0}^{t}[f(\sigma)-f(t)](t-\sigma)^{\mu-3 / 4} d \sigma \\
& =\frac{\Gamma(1 / 4)}{\Gamma(\mu+1 / 4)} f(t) \cdot t^{\mu+1 / 4}+\frac{\Gamma(1 / 4)}{\Gamma(\mu+1 / 4)} \int_{0}^{t}[f(\sigma)-f(t)] \cdot(t-\sigma)^{\mu-3 / 4} d \sigma .
\end{aligned}
$$

This latter term has a zero at $\mu=-1 / 4$ since the integral defines a function analytic for $R e \mu>-1 / 4-\varepsilon$ and $(\Gamma(\mu+1 / 4))^{-1}$ is an entire function with a zero at $\mu=-1 / 4$.

From the identity theorem from 'function theory'

$$
\begin{aligned}
& \frac{1}{\Gamma(\mu)} \int_{0}^{t}\left[\int_{0}^{t} f(\sigma)(t-\sigma)^{-3 / 4} d \sigma-\int_{0}^{\tau} f(\sigma)(\tau-\sigma)^{-3 / 4} d \sigma\right](t-\tau)^{\mu-1} d \tau=F(\mu) \\
& =\frac{t^{\mu}}{\Gamma(\mu+1)} \int_{0}^{t} f(\sigma)(t-\sigma)^{-3 / 4} d \sigma-\frac{\Gamma(1 / 4)}{\Gamma(\mu+1 / 4)} \int_{0}^{t}[f(\sigma)-f(t)](t-\sigma)^{\mu-3 / 4} d \sigma
\end{aligned}
$$




$$
-\frac{\Gamma(1 / 4)}{\Gamma(\mu+5 / 4)} \cdot f(t) \cdot t^{\mu+1 / 4}
$$

for $\Re e \mu>-1 / 4-\varepsilon$. Therefore we find that

$$
\begin{gathered}
\frac{1}{\Gamma(-1 / 4)} \int_{0}^{t}\left[\int_{0}^{t} f(\sigma)(t-\sigma)^{-3 / 4} d \sigma-\int_{0}^{\tau} f(\sigma)(\tau-\sigma)^{-3 / 4} d \sigma\right](t-\tau)^{-5 / 4} d \tau \\
=\frac{t^{-1 / 4}}{\Gamma(3 / 4)} \int_{0}^{t} f(\sigma) \cdot(t-\sigma)^{-3 / 4} d \sigma-\Gamma(1 / 4) \cdot f(t) \\
=-\frac{4 \cdot t^{-1 / 4}}{\Gamma(-1 / 4)} \cdot \int_{0}^{t} f(\sigma) \cdot(t-\sigma)^{-3 / 4} d \sigma-\Gamma(1 / 4) \cdot f(t) .
\end{gathered}
$$

Thus,

$$
\begin{aligned}
{\left[T_{2}\left(T_{1} f\right)\right](t) } & =\frac{-1}{\Gamma(1 / 4) \cdot \Gamma(-1 / 4)}\left\{4 t^{-1 / 4} \cdot \int_{0}^{t} f(\sigma) \cdot(t-\sigma)^{-3 / 4} d \sigma\right. \\
& \left.-4 t^{-1 / 4} \int_{0}^{t} f(\sigma) \cdot(t-\sigma)^{-3 / 4} d \sigma-\Gamma(1 / 4) \cdot \Gamma(1 / 4) f(t)\right\}=f(t) .
\end{aligned}
$$

(ii) All that remains to be shown is that

$$
\sup _{t} t^{\lambda-1 / 4}\left|\left(T_{1} f\right)(t)\right|<+\infty \text {. }
$$

Adding and subtracting $f(t)$ in the integrand we have

$$
\begin{aligned}
& t^{\lambda-1 / 4}\left(T_{1} f\right)(t)=\frac{t^{\lambda-1 / 4}}{\Gamma(1 / 4)} \int_{0}^{t} f(\sigma) \cdot(t-\sigma)^{-3 / 4} d \sigma \\
& \quad=\frac{t^{\lambda-1 / 4}}{\Gamma(1 / 4)} \int_{0}^{t}[f(\sigma)-f(t)] \cdot(t-\sigma)^{-3 / 4} d \sigma+\frac{t^{\lambda-1 / 4}}{\Gamma(1 / 4)} \cdot f(t) \cdot \int_{0}^{t}(t-\sigma)^{-3 / 4} d \sigma .
\end{aligned}
$$

Thus,

$$
\begin{aligned}
& t^{\lambda-1 / 4} \cdot\left|\left(T_{1} f\right)(t)\right| \leq t^{\lambda-1 / 4} \cdot \frac{|f|_{1}}{\Gamma(1 / 4)} \cdot \int_{0}^{t} \sigma^{-\lambda} \cdot(t-\sigma)^{\varepsilon-3 / 4} d \sigma+\frac{4}{\Gamma(1 / 4)} \cdot t^{\lambda} \cdot|f(t)| \\
& \quad=t^{\lambda-1 / 4} \cdot \frac{|f|_{1}}{\Gamma(1 / 4)} \cdot t^{-\lambda+1 / 4+\varepsilon} \cdot \int_{0}^{1} s^{-\lambda} \cdot(1-s)^{\varepsilon-3 / 4} d s+\frac{4}{\Gamma(1 / 4)} \cdot t^{\lambda} \cdot|f(t)| \\
& \quad \leq \text { (constant) } \cdot T^{\varepsilon}|f|_{1}+\frac{4}{\Gamma(1 / 4)}\left[t^{\lambda} \frac{|f(t)-f(T)|](T-t)^{\varepsilon}+\frac{4}{\Gamma(1 / 4)} t^{\lambda}|f(T)|}{|t-T|^{\varepsilon}}\right](\text { constant }) \cdot T^{\varepsilon}|f|_{1}+\frac{4}{\Gamma(1 / 4)} \cdot T^{\lambda}|f(T)|<+\infty .
\end{aligned}
$$

Q.E.D.

THEOREM 3. $g \in S_{2}$

(i) $T_{1} T_{2}=I_{2}$, where $I_{2}$ is the identity transformation on $S_{2}$.

(ii) $T_{2} g \in S_{1}$. 
Proof. Part (i) is proven exactly as part (i) of Theorem 2 and part (ii) follows directly from the definitions of $S_{2}$ and $T_{2}$.

Q.E.D.

Consider the following system of equations made up from the terms with singular kernels in (2.14).

$$
\begin{aligned}
& \frac{1}{2} \phi(t)+\psi(t) \cdot k(0, t)+\int_{0}^{t}[\psi(t)-\psi(\sigma)] \cdot D_{\sigma} k(0, t-\sigma) d \sigma=f(t) \\
& \int_{0}^{t} \phi(\sigma) \cdot D_{y}^{2} k(0, t-\sigma) d \sigma-\frac{1}{2} \psi(t)=g(t)^{3}
\end{aligned}
$$

where $f \in S_{1}$ and $g \in S_{2}$. Now

$$
\begin{aligned}
D_{y}^{2} k(0, t-\sigma) & =\frac{-1}{2 \pi} \int_{-\infty}^{\infty} \xi^{2} \cdot e^{-\xi^{4}(t-\sigma)} d \xi \\
& =\frac{-1}{2 \pi}(t-\sigma)^{-3 / 4} \int_{-\infty}^{\infty} \eta^{2} \cdot e^{-\eta^{4}} d \eta=-\frac{1}{\pi}(t-\sigma)^{-3 / 4} \int_{0}^{\infty} \eta^{2} e^{-\eta^{4}} d \eta \\
& =-\frac{1}{4 \pi}(t-\sigma)^{-3 / 4} \cdot \int_{0}^{\infty} \xi^{-1 / 4} \cdot e^{-\xi} d \xi=\frac{\Gamma(3 / 4)}{4 \pi} \cdot(t-\sigma)^{-3 / 4}
\end{aligned}
$$

Similarly,

$$
D_{\sigma} k(0, t-\sigma)=\frac{\Gamma(5 / 4)}{4 \pi} \cdot(t-\sigma)^{-5 / 4}
$$

and

$$
k(0, t)=\frac{\Gamma(1 / 4)}{4 \pi} \cdot t^{-1 / 4}
$$

Then using the fact that $\Gamma(3 / 4) \cdot \Gamma(1 / 4)=\pi \csc \pi / 4=\sqrt{2} \cdot \pi$

$$
\begin{gathered}
\int_{0}^{t} \phi(\sigma) \cdot D_{\searrow}^{2} k(0, t-\sigma) d \sigma=-\frac{\Gamma(3 / 4)}{4 \pi} \int_{0}^{t} \phi(\sigma) \cdot(t-\sigma)^{-3 / 4} d \sigma \\
=-\frac{\Gamma(3 / 4) \cdot \Gamma(1 / 4)}{4 \pi}\left(T_{1} \phi\right)(t)=-\frac{1}{2 \sqrt{2}}\left(T_{1} \phi\right)(t) .
\end{gathered}
$$

Similarly,

$$
\psi(t) \cdot k(0, t)+\int_{0}^{t}[\psi(t)-\psi(\sigma)] \cdot D_{\sigma} k(0, t-\sigma) d \sigma=\frac{1}{2 \sqrt{2}}\left(T_{2} \psi\right)(t) .
$$

Thus from (3.4) and (3.5) we can write (3.3) as

3 This is just the system of integrals equations one obtains for the problem on the half-space $(0<x<\infty) \otimes(0, T)$. 
$(3.3)^{\prime}$

$$
\left\{\begin{array}{l}
\frac{1}{2} \phi+\frac{1}{2 \sqrt{2}} T_{2} \psi=f \\
-\frac{1}{2 \sqrt{2}} T_{1} \phi-\frac{1}{2} \psi=g .
\end{array}\right.
$$

Using Theorems 2 and 3, we can solve this system of equations by formally applying $T_{1}$ and $T_{2}$. Applying $(1 / \sqrt{2}) T_{2}$ to the second equation and adding to the first gives

$$
-\frac{1}{4} T_{2} T_{1} \phi+\frac{1}{2} \phi=f+\frac{1}{\sqrt{2}} T_{2} g .
$$

Since $T_{2} T_{1}=I_{1}$, we find that

$$
\phi=4\left(f+\frac{1}{\sqrt{2}} T_{2} g\right)=8\left(\frac{1}{2} f+\frac{1}{2 \sqrt{2}} T_{2} g\right) .
$$

Similarly, we find that

$$
\psi=-8\left(\frac{1}{2 \sqrt{2}} T_{1} f+\frac{1}{2} g\right) .
$$

Thus the solution of $(3.3)^{\prime}$ is given by

$$
\left\{\begin{array}{l}
\phi=8\left(\frac{1}{2} f+\frac{1}{2 \sqrt{2}} T_{2} g\right) \\
\psi=-8\left(\frac{1}{2 \sqrt{2}} T_{1} f+\frac{1}{2} g\right) .
\end{array}\right.
$$

Defining

$$
M=\left(\begin{array}{ll}
\frac{1}{2} I_{1} & \frac{1}{2 \sqrt{2}} T_{2} \\
-\frac{1}{2 \sqrt{2}} T_{1} & -\frac{1}{2} I_{2}
\end{array}\right)
$$

where $M$ is an operator on the product $S_{1} \otimes S_{2}$ we can write (3.3)' as $(3.3)^{\prime \prime}$

$$
M \Phi=F
$$

where

$$
\Phi=\left(\begin{array}{l}
\phi \\
\psi
\end{array}\right), \quad F=\left(\begin{array}{l}
f \\
g
\end{array}\right) ;
$$

and (3.6) as

$$
\Phi=8 M F
$$


Thus,

$$
M^{-1}=8 M
$$

Define for suitable functions:

$$
\begin{aligned}
& (S f)(t)=\int_{0}^{t} f(\sigma) \cdot D_{y}^{3} k(1, t-\sigma) d \sigma \\
& (U f)(t)=\int_{0}^{t} f(\sigma) \cdot D_{\sigma} k(1, t-\sigma) d \sigma \\
& (V f)(t)=\int_{0}^{t} f(\sigma) \cdot D_{y}^{2} k(1, t-\sigma) d \sigma
\end{aligned}
$$

In terms of the above defined operators and $T_{1}$, and $T_{2}$, we can write the general system (2.14) as:

$$
\left\{\begin{array}{l}
\frac{1}{2} \phi+S \phi+\frac{1}{2 \sqrt{2}} T_{2} \psi+U \psi=A \\
-\frac{1}{2 \sqrt{2}} T_{1} \phi+V \phi-\frac{1}{2} \psi+S \psi=B
\end{array}\right.
$$

or as,

$$
M \Phi+N \Phi=F \in S_{1} \otimes S_{2}
$$

where

$$
N=\left(\begin{array}{ll}
S & U \\
V & S
\end{array}\right), \quad F=\left(\begin{array}{l}
A \\
B
\end{array}\right)
$$

From (2.2) it follows that all of the kernels in the operators in $N$ are bounded (in fact, they are $C^{\infty}$ functions).

Write $(3.9)^{\prime}$ as

$$
\left(I+N M^{-1}\right) M \Phi=F
$$

where

$$
I=\left(\begin{array}{ll}
I_{1} & 0 \\
0 & I_{2}
\end{array}\right)
$$

is the identity transformation on $S_{1} \otimes S_{2}$ This is certainly meaningful since $N M^{-1}$ is well-defined and likewise $\left(I+N M^{-1}\right) M$.

LEMmA. All of the kernels in the operators in $N M^{-1}$ are bounded and differentiable. 
Proof.

$$
\begin{aligned}
N M^{-1} & =8\left(\begin{array}{ll}
S & U \\
V & S
\end{array}\right)\left(\begin{array}{ll}
\frac{1}{2} I_{1} & \frac{1}{2 \sqrt{2}} T_{2} \\
-\frac{1}{2 \sqrt{2}} T_{1} & -\frac{1}{2} I_{2}
\end{array}\right) \\
& =8\left(\begin{array}{ll}
\frac{1}{2} S-\frac{1}{2 \sqrt{2}} U T_{1} & \frac{1}{2 \sqrt{2}} S T_{2}-\frac{1}{2} U \\
\frac{1}{2} V-\frac{1}{2 \sqrt{2}} S T_{1} & \frac{1}{2 \sqrt{2}} V T_{2}-\frac{1}{2} S
\end{array}\right) .
\end{aligned}
$$

We shall carry out the proof for $S T_{2}$. The proofs of the remaining ones follows exactly the same lines.

For $f \in S_{2}$ :

$\left[S\left(T_{2} f\right)\right](t)$

$$
\begin{aligned}
= & \int_{0}^{t}\left\{\frac{-1}{\Gamma(-1 / 4)}\left[4 \tau^{-1 / 4} \cdot f(\tau)+\int_{0}^{\tau}[f(\tau)-f(\sigma)](\tau-\sigma)^{-5 / 4} d \sigma\right]\right\} D_{y}^{3} k(1, t-\tau) d \tau \\
= & -\frac{4}{\Gamma(-1 / 4)} \int_{0}^{t} \tau^{-1 / 4} \cdot f(\tau) \cdot D_{y}^{3} k(1, t-\tau) d \tau \\
& -\frac{1}{\Gamma(-1 / 4)} \int_{0}^{t}\left(\int_{0}^{t}[f(\tau)-f(\sigma)] \cdot(\tau-\sigma)^{-5 / 4} d \sigma\right) \cdot D_{y}^{3} k(1, t-\tau) d \tau .
\end{aligned}
$$

We proceed as in the proof of Theorem 2. Let

$$
F(\mu)=\frac{1}{\Gamma(\mu)} \int_{0}^{t}\left(\int_{0}^{\tau}[f(\tau)-f(\sigma)] \cdot(\tau-\sigma)^{\mu-1} d \sigma\right) \cdot D_{y}^{3} k(1, t-\tau) d \tau .
$$

This defines an analytic function for $\Re e \mu>-1 / 4$. Now restrict $\mu$ so that $\mathfrak{R e} \mu>0$.

Then

$$
\begin{aligned}
F(\mu)= & \frac{1}{\Gamma(\mu)} \int_{0}^{t} f(\tau) \cdot\left(\int_{0}^{\tau}(\tau-\sigma)^{\mu-1} d \sigma\right) \cdot D_{y}^{3} k(1, t-\tau) d \tau \\
& -\frac{1}{\Gamma(\mu)} \int_{0}^{t}\left(\int_{0}^{\tau} f(\sigma)(\tau-\sigma)^{\mu-1} d \sigma\right) \cdot D_{y}^{3} k(1, t-\tau) d \tau \\
= & \frac{1}{\mu \Gamma(\mu)} \int_{0}^{t} f(\tau) \cdot \tau^{\mu} \cdot D_{y}^{3} k(1, t-\tau) d \tau \\
& -\frac{1}{\Gamma(\mu)} \int_{0}^{t}\left(\int_{0}^{\tau} f(\sigma) \cdot(\tau-\sigma)^{\mu-1} d \sigma\right) \cdot D_{y}^{3} k(1, t-\tau) d \tau .
\end{aligned}
$$

Interchanging the order of integration in the second term gives

$$
\frac{1}{\Gamma(\mu)} \int_{\sigma}^{t} f(\sigma)\left(\int_{0}^{t}(\tau-\sigma)^{\mu-1} \cdot D_{y}^{3} k(1, t-\tau) d \tau\right) d \sigma .
$$


Integrating the inner integral by parts $n$ gives

$$
\frac{1}{\Gamma(\mu+n)} \int_{0}^{t} f(\sigma)\left((-1)^{n} \int_{\sigma}^{t}(\tau-\sigma)^{\mu+n-1} D_{\tau}^{n} D_{y}^{3} k(1, t-\tau) d \tau\right) d \sigma,
$$

which is analytic for $\Re e \mu>-n$.

Thus from the identity theorem we have that

$$
\begin{aligned}
& \frac{1}{\Gamma(\mu)} \int_{0}^{t}\left(\int_{0}^{\tau}[f(\tau)-f(\sigma)] \cdot(\tau-\sigma)^{\mu-1} d \sigma\right) \cdot D_{y}^{3} k(1, t-\tau) d \tau \\
& \quad=F(\mu)=\frac{1}{\mu \Gamma(\mu)} \int_{0}^{t} f(\tau) \cdot \tau^{\mu} \cdot D_{y}^{3} k(1, t-\tau) d \tau \\
& \quad+\frac{(-1)^{n+1}}{\Gamma^{\prime}(\mu+n)} \int_{\sigma}^{t} f(\sigma) \cdot\left(\int_{\sigma}^{t}(\tau-\sigma)^{\mu+n-1} \cdot D_{\tau}^{n} D_{y}^{3} k(1, t-\tau) d \tau\right) d \sigma, \Re e \mu>-1 / 4 .
\end{aligned}
$$

Taking the limit as $\mu \downarrow-1 / 4$, we get

$$
\begin{aligned}
& \frac{1}{\Gamma(-1 / 4)} \int_{0}^{t}\left(\int_{0}^{\tau}[f(\tau)-f(\sigma)] \cdot(\tau-\sigma)^{-5 / 4} d \sigma\right) \cdot D_{y}^{3} k(1, t-\tau) d \tau \\
& =-\frac{4}{\Gamma(-1 / 4)} \int_{0}^{t} f(\tau) \cdot \tau^{-1 / 4} D_{y}^{3} k(1, t-\tau) d \tau \\
& \quad+\frac{(-1)^{n+1}}{\Gamma(n-1 / 4)} \int_{0}^{t} f(\sigma) \cdot\left(\int_{\sigma}^{t}(\tau-\sigma)^{n-5 / 4} \cdot D_{\tau}^{n} D_{y}^{3} k(1, t-\tau) d \tau\right) d \sigma .
\end{aligned}
$$

Hence

$$
\left[S\left(T_{2} f\right)\right](t)=\frac{(-1)^{n}}{\Gamma(n-1 / 4)} \int_{0}^{t} f(\sigma) \cdot\left(\int_{\sigma}^{t}(\tau-\sigma)^{n-5 / 4} \cdot D_{\tau}^{n} D_{y}^{3} k(1, t-\tau) d \tau\right) d \sigma .
$$

From (2.2)

$$
\begin{aligned}
\left|\int_{\sigma}^{t}(\tau-\sigma)^{n-5 / 4} \cdot D_{\tau}^{n} D_{y}^{3} k(1, t-\tau) d \tau\right| & \leq(\text { Constant }) \cdot \int_{\sigma}^{t}(\tau-\sigma)^{n-5 / 4} d \tau \\
& =(\text { Constant }) \cdot(t-\sigma)^{n-1 / 4}
\end{aligned}
$$

Clearly the kernel is continuous and differentiable for $0 \leq \sigma \leq t$. In fact, we could conclude that it is infinitely often differentiable.

Q.E.D.

The above Lemma shows that $I+N M^{-1}$ is essentially a perturbation of the identity. . That is, the problem is reduced to solving a system of Volterra type integral equations with bounded and differentiable kernels.

APPENDIX: Derivation of Estimate (2.2). This appendix is included at the suggestion of the referee in order to make this paper essentially self contained. 
From (2.1)

$$
D_{x}^{n} k(x, t)=(2 \pi)^{-1} \int_{-\infty}^{\infty}(-i z)^{n} \exp \left(-i z x-z^{4} t\right) d z, \quad t>0 .
$$

Making the change of variable $z t^{1 / 4}=y$ gives

$$
D_{x}^{n} k(x, t)=(-i)^{n}(2 \pi)^{-1} t^{-(1+n) / 4} \int_{-\infty}^{\infty} y^{n} \exp \left(-i y x t^{-1 / 4}-y^{4}\right) d y .
$$

The integral in (1) considered as an integral in the complex plane is easily seen to be equal to

$$
\int_{-\infty}^{\infty}(y+i c)^{n} \exp \left[-i a^{3}(y+i c)-(y+i c)^{4}\right] d y
$$

where $c$ is any real number and $a=\left(x t^{-1 / 4}\right)^{1 / 3}$.

Denoting the integral (2) by $I$ we find upon expanding that

$$
\begin{aligned}
I= & \left(\exp \left[a^{3} c-c^{4}\right]\right) \sum_{j=0}^{n}\left(\begin{array}{c}
n \\
j
\end{array}\right)(i c)^{n-j} \\
& \times \int_{-\infty}^{\infty} y^{j} \exp \left[-i\left(a^{3} y+4 y^{3} c-4 y c^{3}\right)-\left(y^{4}-6 y^{2} c^{2}\right)\right] d y .
\end{aligned}
$$

Using the inequality $6 y^{2} c^{2} \leq 9 R^{-1} y^{4}+R c^{4}$ with $R>9$ it follows that

$$
|I| \leq\left(\exp \left[a^{3} c+(R-1) c^{4}\right]\right) \sum_{j=0}^{n}\left(\begin{array}{c}
n \\
j
\end{array}\right)|c|^{n-j} \int_{-\infty}^{\infty}|y|^{j} \exp \left[-y^{4}\left(1-9 R^{-1}\right)\right] d y .
$$

Setting

$$
A(n)=\max _{0 \leq j \leq n}\left\{\int_{-\infty}^{\infty}|y|^{j} \exp \left[-y^{4}\left(1-9 R^{-1}\right)\right] d y\right\}
$$

we obtain the inequality

$$
|I| \leq A(n)(1+|c|)^{n} \exp \left[a^{3} c+(R-1) c^{4}\right] .
$$

Now choose $c=-\mu(R-1)^{-1 / 3} a, 0<\mu<1$. Then

$$
a^{3} c+(R-1) c^{4}=-\mu\left(1-\mu^{3}\right)(R-1)^{-1 / 3} a^{4}<0
$$

and

$$
|I| \leq A(n)\left[1+\mu(R-1)^{-1 / 3}|a|\right]^{n} \exp \left[-\mu\left(1-\mu^{3}\right)(R-1)^{-1 / 3} a^{4}\right] .
$$

Setting

$$
B(n)=\left\{\max _{z \geq 0}\right\}\left[1+\mu(R-1)^{-1 / 3} z\right]^{n} \exp \left[-2^{-1} \mu\left(1-\mu^{2}\right)(R-1)^{-1 / 3} z^{4}\right]
$$

and replacing $a$ by $\left(x t^{-1 / 4}\right)^{1 / 3}$ we get the inequality

$$
|I| \leq A(n) B(n) \exp \left[-2^{-1} \mu\left(1-\mu^{3}\right)(R-1)^{-1 / 3}\left(x^{4} t^{-1}\right)^{1 / 3}\right] .
$$


Estimate (2.2) is obtained from (1), (2), and (3) with

$$
C_{1}=(2 \pi)^{-1} A(n) B(n) \text { and } C_{2}=2^{-1} \mu\left(1-\mu^{3}\right)(R-1)^{-1 / 3} \text {. }
$$

Acknowledgement. This paper is based on the first part of the author's dissertation written at the University of Minnesota under the direction of Professor A. N. Milgram. The author wishes to express his appreciation to Professor Milgram for his guidance.

\section{BIBLIOGRAPHY}

1. M. Gevrey, Sur les équations aux dérivées partielles du type parabolique, Journal de Mathématiques, (6), 10 (1913), 105-148.

2. P. G. Kirmser, Thesis, University of Minnesota.

3. O. A. Ladyzhenskaya, On the uniqueness of the solution of the Cauchy problem for a linear parabolic equation, Matematiceskii Sbornik, 27, 69 (1950), 175-184.

4. M. Riesz, L'integrale de Riemann-Liouville et le problème de Cauchy, Acta Mathematica 81 (1949), 1-223.

5. P. C. Rosenbloom, Linear equations of parabolic type with constant coefficients, Contributions to the Theory of Partial Differential Equations, Princeton University Press, Princeton (1954), 191-200.

6. A. Tykhonov, Sur l'equation de la chaleur à plusieurs variables, Moscow Universitet Bulletin, Série international, Section A, Mathématiques et Mécanique, 1, No. 9, (1938), 1-44. 7. P. K. Zaragiya, On the solution of boundary problems for equations of parabolic type by the method of potentials, Soobšč. Akad. Nauk Gruzin, SSR, 15, (1954) 569-573. 


\section{PACIFIC JOURNAL OF MATHEMATICS}

\section{EDITORS}

\author{
David Gilbarg \\ Stanford University \\ Stanford, California \\ F. H. Brownell \\ University of Washington \\ Seattle 5 , Washington
}

\author{
A. L. Whiteman \\ University of Southern California \\ Los Angeles 7, California \\ L. J. PAIGE \\ University of California \\ Los Angeles 24, California
}

\section{ASSOCIATE EDITORS}

\author{
E. F. BECKENBACH \\ T. M. CHERRY \\ D. DERRY
}
E. HEWITT
A. HORN
L. NACHBIN
M. OHTSUKA
H. L. ROYDEN
M. M. SCHIFFER

E. SPANIER

E. G. STRAUS

F. WOLF

\section{SUPPORTING INSTITUTIONS}

\author{
UNIVERSITY OF BRITISH COLUMBIA \\ CALIFORNIA INSTITUTE OF TECHNOLOGY \\ UNIVERSITY OF CALIFORNIA \\ MONTANA STATE UNIVERSITY \\ UNIVERSITY OF NEVADA \\ NEW MEXICO STATE UNIVERSITY \\ OREGON STATE COLLEGE \\ UNIVERSITY OF OREGON \\ OSAKA UNIVERSITY \\ UNIVERSITY OF SOUTHERN CALIFORNIA
}

\author{
STANFORD UNIVERSITY \\ UNIVERSITY OF TOKYO \\ UNIVERSITY OF UTAH \\ WASHINGTON STATE COLLEGE \\ UNIVERSITY OF WASHINGTON

AMERICAN MATHEMATICAL SOCIETY
CALIFORNIA RESEARCH CORPORATION
HUGHES AIRCRAFT COMPANY
SPACE TECHNOLOGY LABORATORIES
NAVAL ORDNANCE TEST STATION

Mathematical papers intended for publication in the Pacific Journal of Mathematics should be typewritten (double spaced), and the author should keep a complete copy. Manuscripts may be sent to any one of the four editors. All other communications to the editors should be addressed to the managing editor, L. J. Paige at the University of California, Los Angeles 24, California. ..

50 reprints per author of each article are furnished free of charge; additional copies may be obtained at cost in multiples of 50 .

The Pacific Journal of Mathematics is published quarterly, in March, June, September, and December. The price per volume (4 numbers) is $\$ 12.00$; single issues, $\$ 3.50$. Back numbers are available. Special price to individual faculty members of supporting institutions and to individual members of the American Mathematical Society: $\$ 4.00$ per volume; single issues, $\$ 1.25$.

Subscriptions, orders for back numbers, and changes of address should be sent to Pacific Journal of Mathematics, 2120 Oxford Street, Berkeley 4, California.

Printed at Kokusai Bunken Insatsusha (International Academic Printing Co., Ltd.), No. $6_{4}$ 2-chome, Fujimi-cho, Chiyoda-ku, Tokyo, Japan.

PUBLISHED BY PACIFIC JOURNAL OF MATHEMATICS, A NON-PROFIT CORPORATION

The Supporting Institutions listed above contribute to the cost of publication of this Journăl, but they are not owners or publishers and have no responsibility for its content or policies. 


\section{Pacific Journal of Mathematics}

\section{Vol. 10, No. $3 \quad$ November, 1960}

Glen Earl Baxter, An analytic problem whose solution follows from a simple

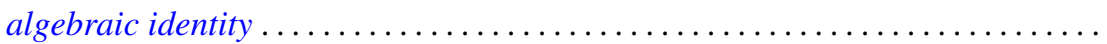

Leonard D. Berkovitz and Melvin Dresher, A multimove infinite game with linear payoff. .

Earl Robert Berkson, Sequel to a paper of A. E. Taylor ......................

Gerald Berman and Robert Jerome Silverman, Embedding of algebraic systems.... 767

Peter Crawley, Lattices whose congruences form a boolean algebra . . . . . ...... 777

Robert E. Edwards, Integral bases in inductive limit spaces . . . . . . . . . . . . . . .

Daniel T. Finkbeiner, II, Irreducible congruence relations on lattices . . . . . . . . . .

William James Firey, Isoperimetric ratios of Reuleaux polygons . . . . . . . . . . . 787

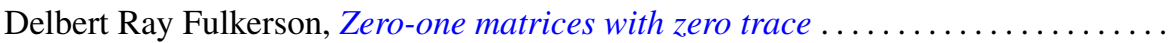

Leon W. Green, A sphere characterization related to Blaschke's conjecture........

Israel (Yitzchak) Nathan Herstein and Erwin Kleinfeld, Lie mappings in

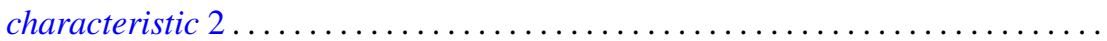

Charles Ray Hobby, A characteristic subgroup of a $p$-group .................

R. K. Juberg, On the Dirichlet problem for certain higher order parabolic

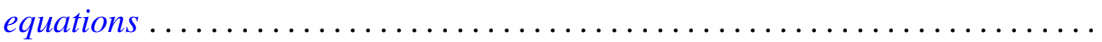

Melvin Katz, Infinitely repeatable games ......................

Emma Lehmer, On Jacobi functions . . . . . . . . . . . . . . . . . . . . . . . . .

D. H. Lehmer, Power character matrices

Henry B. Mann, A refinement of the fundamental theorem on the density of the sum of two sets of integers.

Marvin David Marcus and Roy Westwick, Linear maps on skew symmetric matrices: the invariance of elementary symmetric functions . .

Richard Dean Mayer and Richard Scott Pierce, Boolean algebras with ordered

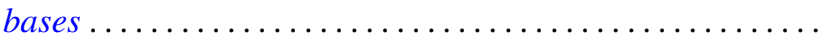

Trevor James McMinn, On the line segments of a convex surface in $E_{3} \ldots$

Frank Albert Raymond, The end point compactification of manifolds ..

Edgar Reich and S. E. Warschawski, On canonical conformal maps of regions of arbitrary connectivity

Marvin Rosenblum, The absolute continuity of Toeplitz's matrices...

Lee Albert Rubel, Maximal means and Tauberian theorems . .

Helmut Heinrich Schaefer, Some spectral properties of positive linear operators

Jeremiah Milton Stark, Minimum problems in the theory of pseudo-conformal transformations and their application to estimation of the curvature of the invariant metric.

Robert Steinberg, The simplicity of certain groups ...

Hisahiro Tamano, On paracompactness. .

Angus E. Taylor, Mittag-Leffler expansions and spectral theory .

Marion Franklin Tinsley, Permanents of cyclic matrices ...... . 